\title{
Clinical Applications of Pulsed Low Dose-Rate Radiation Therapy
}

\author{
Lili Chen
}

Department of Radiation Oncology, Fox Chase Cancer Center, Philadelphia, PA 19111, USA

\section{ABSTRACT}

Pulsed low dose-rate radiotherapy (PLDR) is a novel radiotherapy technique for cancer treatments, which relies on two radiobiological findings, the hyper-radiosensitivity of tumor cells at small doses and the reduced normal tissue toxicity at low dose rates. PLDR delivers the daily radiation dose in a number of sub-fractions (pulses) with a preset time interval to achieve an effective low dose rate. PLDR can be delivered on existing clinical machines using different treatment optimization strategies and delivery techniques. Clinical trials have been carried out to study the application of PLDR for various cancers especially for recurrent cancers. Preliminary results from these clinical studies have shown favorable outcome. This paper briefly describes the PLDR technique, the planning requirements and its clinical applications in cancer treatment.

KEYWORDS: External beam radiotherapy (EBRT); Dose rate effect; Hyper-radiosensitivity; Tumor control; Normal tissue toxicity; Pulsed low dose-rate radiotherapy (PLDR); Transition dose

\section{INTRODUCTION}

There have been tremendous advancements in radiation therapy (RT) in the last several decades especially with the development of computer controlled linear accelerators and multi-leaf collimators (MLC), treatment planning systems and optimization techniques, and various imaging modalities and treatment targeting/guidance techniques. This is marked by the superior dose distributions and dosimetry accuracy available with many advanced treatment machines, which have resulted in improved tumor control through target dose escalation and better quality of life due to reduced normal tissue toxicities and treatment time/complexities.

Advanced RT equipment and treatment techniques have been employed to explore various dose and fractionation schemes. Intensity modulated radiotherapy (IMRT) and volumetric modulated arc therapy (VMAT) have provided superior dose distributions to allow for dose escalation and hypofractionation trials, which require better radiobiological understanding. For example, the prescription dose to the target and the dosimetric constraints for the critical structures have been a major concern in the design of clinical trials for stereotactic body radiation therapy (SBRT), which delivers a few treatment fractions of very large doses [14]. Proton and carbon ion therapy has also garnered more interests both

\section{Vol No: 06, Issue: 01}

Received Date: March 16, 2021

Published Date: May 21, 2021

\section{*Corresponding Author}

\section{Lili Chen}

Department of Radiation Oncology, Fox Chase Cancer Center, Philadelphia, PA 19111, USA

E-mail: lili.chen@fccc.edu

Citation: Chen L. (2021). Clinical Applications of Pulsed LowDose-Rate Radiation Therapy. Mathews J Cancer Sci. (6)1:27.

Copyright: Chen L (C) (2021). This is an open-access article distributed under the terms of the Creative Commons Attribution License, which permits unrestricted use, distribution, and reproduction in any medium, provided the original author and source are credited. 
in the US and worldwide [5]. Recent developments of pulsed low-dose rate radiotherapy (PLDR) [6-10] and flash therapy [11-13] are also good examples of RT advancements based on better radiobiological understanding and/or novel RT equipment and techniques.

PLDR is a novel external beam RT delivery technique. The idea behind the PLDR technique is to take advantage of both the hyper-radiosensitivity of tumor cells below their transition doses, which are generally greater than those of normal tissues, and the increased normal tissue repair at low dose rates $[6,9]$. The way to achieve this is to divide a daily radiotherapy treatment into a number of subfractions (pulses) with each subfractional dose less than the tumor transition dose but greater than the normal tissue transition dose so that the radiation repair is triggered in normal tissues but not in tumor cells. The radiation pulses are delivered at certain intervals to achieve an effective low dose rate to maximize the normal tissue repair process.

PLDR treatment was initiated with simple treatment techniques using single field electron beams or 3D conformal photon beams [6-8]. Advanced delivery systems and treatment planning optimization strategies were developed to support re-irradiation protocols [10,14-20]. A number of clinical trials have been carried out to study the potential of PLDR treatments for recurrent cancers, bulky and/or radiation resistant cancers and the combination of PLDR with other treatment modalities, e.g. chemotherapy [7,8,20-27]. Preliminary clinical results of PLDR have shown favorable disease control and acceptable normal tissue toxicities for many recurrent cancers.

This paper will briefly describe the PLDR technique and the dosimetric requirements for PLDR treatment planning and advanced beam delivery techniques such as IMRT and VMAT. The clinical applications of the PLDR technique for cancer treatment will be reviewed based on the treatment site including brain, head and neck, breast, thorax and pelvis.

\section{THE PLDR TECHNIQUE}

Low-dose hyper-radiosensitivity (LDHRS) has been found in many cell lines of both tumor and some normal tissues, as well as in human metastatic tumors [28-36]. LDHRS was not observed in the intrinsically radiosensitive cell lines, whereas radioresistant cell lines demonstrated the most marked LDHRS [37,38]. A possible explanation for LDHRS is the lack of DNA repair below a given threshold dose (transition dose), which is cell-type dependent and has been typically observed in the dose range of 0.2 Gy to $0.6 \mathrm{~Gy}$
[28-42]. In contrary to normal tissue sparing due to repair of sublethal DNA damage during low dose-rate irradiation, increased radiosensitivity of tumor cells was observed when the dose-rate was decreased. This so-called inverse doserate effect [28-30] can be observed at dose-rates below 1 Gy/h in cells showing LDHRS [43-45].

The dose rate effect in radiobiology and radiation therapy has been investigated extensively but the mechanisms of radiation damage repair for mammalian cells are still not fully understood at the molecular level $[46,47]$. The dose rate effect on DNA repair is most dramatic between 0.01 and 1 Gy/min [47]. Generally, increased DNA repair will occur over a prolonged treatment time for either single-strand or double-strand breaks, resulting in a lower biologically effective dose (BED). At doses above the transition dose [48], which is generally lower for normal tissues than for tumor cells, increased DNA repair will compromise tumor control and further reduce normal tissue damage. Moreover, slowly proliferating normal tissues may be relatively insensitive to low-dose rate irradiation as more sub-lethal damage is repaired prior to cell division than in malignant cells during a protracted treatment session.

Although low-dose-rate external beam radiotherapy (EBRT) was explored many years ago on earlier machines with low dose rates [49-52] the PLDR technique is a novel delivery technique, which can be delivered using commonly available clinical accelerators using both simple delivery techniques such as 2D conventional and 3D conformal therapy (3DCRT) or advanced techniques such as intensitymodulated radiation therapy (IMRT) and volumetricmodulated radiotherapy (VMAT). In order to take advantage of both the low-dose hyper-radiosensitivity for tumors and the increased normal tissue repair at low dose rates, PLDR is designed to deliver the total daily dose in sub-fractions (pulses) of small doses within a limited time frame to achieve an effective low dose rate. For example, a daily dose of $2 \mathrm{~Gy}$ can be delivered in 10 sub-fractions (i.e., pulses) with $3 \mathrm{~min}$ time internals, resulting in an effective dose rate of $0.067 \mathrm{~Gy} /$ min. A theoretical analysis of the tumor control probability (TCP) for PLDR with a pulsed dose scheme was presented by Tome and Howard [6]. Clearly the daily dose of PLDR can be varied for different treatment intent (curative or palliation) or in combination with other therapies (chemotherapy or immunotherapy), e.g. $1.8 \mathrm{~Gy} /$ day in either 9 or 10 subfractions, or two treatments per day at $1.2 \mathrm{~Gy}$ per treatment with 6 sub-fractions. 
Caution must be exercised with the initial introduction of this technique to each body site since not all tumor cells exhibit HRS whereas some normal tissue cells also show increased response to low doses. Fundamentally, it is the improvement in the therapeutic ratio, not just HRS that makes the PLDR technique a treatment modality of choice for many cancers [9]. The rich in-vitro and in-vivo experimental results have laid a strong radiobiological foundation for the PLDR technique, which has also been used to guide pilot studies to find optimal doses, dose rates and fractionation schemes for particular body sites. A clear advantage of the PLDR technique over conventional radiotherapy is the reduced normal tissue damage at lower dose rates, which has offered hope for some recurrent patients with potential severe and/ or life threatening symptoms, who have been otherwise considered unsuitable for re-irradiation treatments with conventional radiotherapy. The fact that many radioresistant tumor cells exhibit higher RHS/IRR ratios at lower doses and dose rates also suggests that the PLDR technique may be a better choice than conventional radiotherapy for some recurrent cancers because of the possible existence of such radioresistant tumor cells. Radiation therapy has experienced the greatest change in the last several decades because of the technological advancements. The improvement in radiobiological understanding has for a long time lagged behind the technological development but it is expected to play a more significant role in the further advancement of radiotherapy for both curative and palliative care.

It is interesting to mention that PLDR was also termed "pulsed reduced dose-rate radiotherapy (PRDR)" in other publications [6-9]. The argument to use "reduced dose rate" rather than "low dose rate" was to avoid confusion with the traditional dose rate definition in brachytherapy [9]. For example, ICRU report 38 "Dose and Volume Specifications for Reporting Intracavitary Therapy in Gynecology" defined low dose rate (LDR): 0.4 - 2 Gy/hour, medium dose rate (MDR): 2 - 12 Gy/hour, and high dose rate (HDR): 12 Gy/ hour and higher [53]. However, the historical development of EBRT did not follow the same dose rate definition as in brachytherapy. For example, traditional linac-based EBRT uses dose rates similar to or higher than that of HDR but has never referred it to "high dose rate" radiotherapy. In a 1991 paper by Hall and Brenner, the range of "low dose rate" was defined as 0.1 - $10 \mathrm{~Gy} /$ hour for radiotherapy and radiobiology [31]. Pierquin $B$, et al. and others were first referred to pioneers of PRDR by Tome and Howard [6] even though all of these original authors actually used the term "low dose rate" RT in their studies [49-52]. The term PLDR was first used by Ma CM, et al. [10], in which the dosimetric requirements for delivering pulsed VMAT were discussed in detail. It was considered to be more definitive and descriptive to use PLDR than PRDR because "reduced dose rate" does not indicate the actual dose-rate range achievable by this special delivery technique. More studies focusing on the strategy for PLDR treatment planning and dose constraints for PLDR plan optimization following the same methodology were reported [15-18]. Many in vitro and in vivo experiments on PLDR were reported to investigate the therapeutic ratio and mechanisms of PLDR, and most of them used the same terminology [54-65]. For the same reason, the term PLDR is used in this paper instead of PRDR although both terms were included in the keywords for the literature research of clinical studies of this treatment technique.

\section{PLDR DOSIMETRY REQUIREMENTS}

A PLDR treatment can be easily delivered on regular clinical accelerators using conventional techniques such as singlefield irradiation and 3D conformal radiotherapy (3DCRT). Early clinical trials for recurrent glioma cancer [7] and breast cancer [8] employed high-energy photon and electron beams from conventional clinical accelerators. Both the dose and dose rate constraints for PLDR are achievable with a single-field photon or electron beam [6]. One can divide the total MUs for the daily 2Gy treatment by 10 sub-fractions with a 3- minute interval (using a stopwatch). The maximum dose difference within the target volume for a single-beam irradiation is typically less than $30 \%$ from the prescription dose (to the centroid of the target volume) mainly due to beam attenuation along the depth direction and electron scattering at air/tissue/bone interfaces, which is unlikely to exceed the transition dose for most tumor tissues (e.g., 0.5Gy). For 3DCRT, one can arrange the beam weights in such a way that the total MUs can be divided into 10 subfractions of $0.2 \mathrm{~Gy}$ each so that the 3 min time interval can be kept between the sub-fractions for the same radiation field/ port or among different fields/ports.

IMRT techniques were explored for PLDR delivery with difficulties $[66,67]$ especially if the entire IMRT plan was delivered within the 3-min interval [14]. The group at Fox Chase Cancer Center published a series of papers reporting on their methods to deliver PLDR using IMRT and VMAT techniques. Ma CM, et al [68] summarized treatment planning strategies/guidelines for IMRT and VMAT as follows: (1) to use 10 beam angles for IMRT and 10 arcs for VMAT (2arc plan to be delivered 5 times) with each angle/arc as a pulse; (2) to set the mean dose (0.2Gy) and maximum dose 
(0.4Gy) to the target per pulse as hard constraints (no hard constraints to the OAR to reduce intensity modulation inside the target volume); (3) to select optimal beam/arc angles to avoid OARs as much as possible; and (4) to add reference structures in or around target/OARs to reduce maximum dose to the target and OARs. IMRT, VMAT and 3DCRT plans were compared for 60 patients to demonstrate the feasibility of IMRT and VMAT for PLDR treatment with superior target dose conformity and critical structure sparing.

\section{CLINICAL INVESTIGATIONS}

Local tumor progression after prior radiation therapy represents a significant therapeutic challenge due to the appropriate concern of unacceptable toxicity to surrounding normal tissues with additional radiation. PLDR is an irradiation strategy that may overcome the limitations of conventional radiation therapy in the setting of large volume recurrent tumors and provides a similar, if not better, level of tumor control when compared to existing treatment options. This section briefly describes the clinical applications of PLDR for treating recurrent cancers and for other clinical potentials.

\section{PRDR for brain cancer}

The first PLDR treatment was reported by Cannon et al for treating recurrent glioma [69] following the dosimetric analysis by Tome and Howard [6]. The patient was initially diagnosed with a Grade II astrocytoma and underwent partial resection followed by RT (54 Gy, $1.8 \mathrm{~Gy} /$ fraction). Three years later, the disease progressed and transformed to a Grade IV astrocytoma. The patient was treated with a near total resection and chemotherapy with minimal response. Then, he was offered PLDR 3DCRT treatment (50 Gy, 2.0 Gy/ fraction, effective dose rate $0.0667 \mathrm{~Gy} / \mathrm{min}$ ). Post treatment MRI demonstrated regression of nodular enhancement and improvement in mass effect and the patient's weakness and seizure activity improved. Despite delivering 104 Gy to the tumor bed and the surrounding brain parenchyma, at no time was there radiographic evidence of radiation-induced normal tissue necrosis. Adkison JB, et al. [7] presented a large retrospective review of PRDR for recurrent glioma patients at the same center. PLDR was delivered using the same regimen to a median dose of 50Gy (range, 20-60 Gy) in 1.8-2.0 Gy/fraction. The mean treatment volume was 403.5 $\pm 189.4 \mathrm{~cm}^{3}$ according to $\mathrm{T} 2$-weighted magnetic resonance imaging and a 2 -cm margin. Their results showed that PLDR allowed for safe retreatment of larger tumor volumes to high doses (average cumulative dose $106.8 \mathrm{~Gy}$ ) with palliative benefit. Patients who received PLDR within 14 months of the first irradiation $(n=43)$ had a median survival of 21 weeks. Those treated $\geq 14$ months after the first RT treatment had a median survival of 28 weeks $(n=43 ; p=0.004$ and $H R=$ 1.82 with a $95 \%$ CI ranging from 1.25 to 3.10 ). According to initial histologic findings, median survival since diagnosis and retreatment was 6.3 years and 11.4 months for lowgrade, 4.1 years and 5.6 months for Grade 3, and 1.6 years and 5.1 months for Grade 4 tumors, respectively. Age at the initial diagnosis, initial low-grade disease, and Karnofsky performance score of $\geq 80$ were shown to be significant predictors of survival after the initiation of PLDR. Previous therapies such as gross vs. subtotal initial resection and systemic therapies (including temozolomide for Grade 4 patients) did not influence survival after PLDR.

The same institution also investigated the clinical potential of PLDR for various re-treatment scenarios. Mohindra et al [21] reported five pediatric and young adult patients with recurrent ependymoma including eight PLDR treatments for two intracranial sites and six spinal locations at a median re-irradiation dose of 40 Gy (range: 30.6-54 Gy). The cumulative radiation doses per site ranged from 90Gy-162.4 Gy with median volume of $882 \mathrm{~cm}^{3}$ encompassed by the $50 \%$ isodose line. At a median post-PLDR follow-up of 64 months, estimated 4-year overall survival and progression-free survival after PLDR was $60 \%$ and $35.7 \%$, respectively. None of the 5 patients developed necrosis on serial MRI scans, and only one patient had progressive mild radiculopathy. Magnuson et al [22] evaluated PLDR re-irradiation as salvage therapy for 23 recurrent GBM patients (after standard radiotherapy/temozolomide) treated with bevacizumab (10 mg/kg) every 2 weeks until progression (median age 53 years; median KPS 80; median progression free survival on bevacizumab 3.7 months). Within 7-14 days of progression on bevacizumab, patients were treated with PLDR to a dose of $54 \mathrm{~Gy}$ in 27 fractions to a median PTV of $424 \mathrm{~cm}^{3}$ along with 2 cycles of concurrent bevacizumab to prevent radiation necrosis. The median overall survival and 6-month overall survival after bevacizumab failure was 6.9 months and $65 \%$, respectively. PLDR re-treatment was well tolerated with no symptomatic grade 3-4 toxicities. They concluded that PLDR may present an excellent treatment option for large recurrent tumors that are not amenable to SRS/SBRT. Witt JS, et al [26] conducted a retrospective study on PLDR re-treatment of recurrent meningioma. Eight patients with recurrent intracranial meningioma were included in this study. Median time between radiation courses was 7.7 years. Median PLDR dose was 54 Gy in 27 fractions to a median target volume of $261.6 \mathrm{~cm}^{3}$. Two patients had in field failure 
with a median follow up of 23.3 months. Progression-free survival at 6 months was $100 \%$. Seven patients were still alive at last follow up. No patient experienced grade 2 or higher acute or late toxicities.

PLDR re-irradiation has been performed by other clinical investigators. Li GH, et al. [70] reported a case study to treat a metastatic lesion in the left occipital lobe for a patient with NSCLC. After initial chemoradiotherapy to the left inferior lung and mediastinal lymph nodes for stage T2N3M0 squamous cell carcinoma, the patient received 40 Gy WBRT for multiple metastatic brain lesions with a 20 Gy boost to a left occipital metastatic site, and later salvage SRS of another 14 Gy in 4 fractions due to persistence of this lesion. Following subsequent recurrence, he received additional SRS of 28 Gy in 8 fractions with concurrent nimustine (ACNU). After the third recurrence, the patient refused surgery and was treated with 3DCRT PLDR of 60 Gy in 30 fractions of 2.0 Gy. The patient had resolution of headache, vomiting and dizziness and continued to live independently with intact cognitive function following PLDR re-treatment. Despite a total of 162 Gy to the left occipital lobe metastasis, the patient had no apparent acute or late neurologic toxicities.

Other investigators have evaluated PLDR re-treatment for recurrent brain tumors through a retrospective study. Murphy ES, et al. [19] reviewed outcomes and toxicities of 24 patients with recurrent CNS tumors mostly treated with 7-field IMRT to a median prescription dose of 54 Gy to a mean PTV of $369 \mathrm{~cm}^{3}$. As discussed previously, no control of the maximum dose per field was considered and the time interval was arranged individually for patients to achieve a $0.067 \mathrm{~Gy} / \mathrm{min}$ effective dose rate. The median progressionfree survival and 6-month progression-free survival after PLDR was 3.1 months and 31\%, respectively. The median overall survival and 6-month overall survival after PLDR was 8.7 months and 71\%, respectively. Fifty percent of patients had $\geq 4$ chemotherapy regimens before the re-treatment. Toxicity was similar to initial treatment, including no cases of radiation necrosis.

A more recent PLDR re-treatment respective study was reported by Bovi JA, et al. [71] who investigated whether the addition of PLDR to bevacizumab improves survival over bevacizumab alone for recurrent high-grade glioma. In this study; 47 patients received bevacizumab monotherapy, and 33 patients received PLDR with bevacizumab. No details were given of the treatment techniques for the PLDR treatment. The total PLDR prescribed dose was 50 to $54 \mathrm{~Gy}$ (in $2 \mathrm{~Gy} /$ fraction with 10 pulsed doses of $0.2 \mathrm{~Gy}$ every 3 minutes for an effective dose rate of $0.067 \mathrm{~Gy} / \mathrm{min}$ ). Their results showed that significant $(\mathrm{P}<.05)$ advantages in progression-free survival (12 vs 4 months; hazard ratio $=2.37$ ) and overall survival (16 vs. 9 months; hazard ratio = 1.68) were observed with PLDR/bevacizumab compared with bevacizumab alone. They suggested further exploration of appropriate patient selection, optimal delivery technique, benefits of concurrent systemic therapies, and possible PLDR-related toxicities.

\section{PRDR for head and neck cancer}

A case report was presented by Li GH, et al. [72] on PLDR re-treatment for a 56-year male with recurrent poorly differentiated squamous cell carcinoma of the nasopharynx metastatic to a cervical lymph node. After an initial diagnosis of T2N2M0 tumor, the patient received 70 Gy in 2Gy/fraction to the gross tumor volume and a metastatic lymph node, and $\geq 50$ Gy to the bilateral cervical lymphatics. Five years later, he received an additional 60 Gy in 2 Gy/fraction followed by surgical dissection for recurrent metastatic lymph nodes in the neck. This was followed by adjuvant chemotherapy with paclitaxel and cisplatin. A second recurrence resistant to cisplatin/fluorouracil and cisplatin/paclitaxel systemic treatments was discovered a year later, and was treated with PLDR 3DCRT (70 Gy in 2 Gy/fraction) and concurrent cetuximab. In total, the patient received up to $190 \mathrm{~Gy}$ to the recurrence region. The recurrent lesion of this patient had a complete response with no apparent radiation-induced normal tissue complications (the patient only experienced Grade 1 acute skin toxicity).

Tong X, et al. [73] reported their initial PLDR re-irradiation experience of 13 patients with recurrent cancers. Among them, 4 patients were treated for recurrent head and neck cancers including one thyroid and 3 neck nodes. The patients received $60-66$ Gy in their previous treatments and the recurrent tumors were treated to 16 - $60 \mathrm{~Gy}$, all in $2 \mathrm{~Gy} /$ fraction. Multiple-field 3DCRT was used with an effective dose rate of $0.067 \mathrm{~Gy} / \mathrm{min}$. The interval between the previous RT and re-irradiation was 13 - 336 months, and the follow-up time was up to 27 months. Their results showed favorable response to the PLDR re-treatment with 1 complete response (CR) and 3 partial responses (PR). Acute toxicities included 2 xerostomia (grade 1) and 2 mucositis (grade 2). Late toxicities included 1 skin fibrosis (grade 2), 2 skin fibrosis (grade 3), 3 hyperpigmentation (grade 2) and 3 xerostomia (grade 1). 


\section{PLDR for breast cancer}

PRDR has shown favorable outcome for recurrent breast cancer as reported by Richards GM, et al. [8]. This early PLDR trial included 17 patients, who previously received postmastectomy radiation treatment with a median prior dose of 60 Gy. All patients were treated using conventional conformal techniques to a median dose of 54 Gy in 2 Gy/ fraction. The treatment sites included the chest well, axilla and/or supraclavicular region and the median treatment volume was 2,084 $\mathrm{cm}^{3}$. Eight patients received concurrent chemotherapy. Post PLDR results showed that 15 patients had complete resolution of the loco-regional recurrence with an estimated 2-y local control rate of $92 \%$ at a median follow-up of 18 months and a 23\% grade 3 acute skin toxicity. Only two patients developed Grade 3 and Grade 4 non-healing chest wall ulcers and none of the patients, who received treatment to the axilla and/or supraclavicular area, experienced brachial plexopathy.

A follow-up review was performed by Burr AR, et al. [27] that also included 26 additional patients treated with PLDR using both conventional conformal techniques and step-andshoot IMRT between 2000 and 2018. The complete response rate was $83.3 \%$ in 24 treated patients with gross disease. Locoregional recurrence-free survival was $81.3 \%$ and $73.8 \%$ for all patients at 1 and 2 years, respectively. Overall survival for patients with localized disease was $95.7 \%$ at 1 year and $91.1 \%$ at 2 years. The rate of acute grade 3 radiation dermatitis was $25.6 \%$ with no other acute grade 3 toxicities. Grade 3 late toxicity occurred in $18.6 \%$ of patients. They concluded that PLDR with capecitabine was a well-tolerated and effective method for treating patients with recurrent breast cancer. Prospective studies are necessary to compare side effects and efficacy with conventional dose rate reirradiation and to evaluate the potential role for capecitabine in the recurrent setting.

\section{PLDR for thoracic cancer}

The Fox Chase group was the first to investigate advanced delivery techniques systematically for PLDR treatment of recurrent cancers to further reduce normal tissue toxicities [10,15-17]. These delivery techniques were used in their phase I dose escalation trial to investigate PLDR irradiation for palliation of recurrent tumors [74]. Meyer JE, et al. [75] reported the outcome of 16 patients enrolled between 2011 and 2015. Five patients received 40 Gy, three received 50 Gy and 8 received 60 Gy in 2 Gy/fraction. No DLTs were recorded. Median follow-up was 10.2 months (range: 1.840). Disease assessment was performed in 15 patients. Best response was 1 complete response (7\%), 2 partial response (13\%) and 6 stable disease (40\%). Median progressionfree survival (PFS) was 4.4 months (95\% CI: 2.1-11.6). The first site of progression was outside the treated region in all patients. Overall survival (OS) was 14.3 months (95\% CI: 6.7-undefined).

A follow-up study was presented by Lee CT, et al. [23] on the efficacy and toxicity of PLDR re-treatment for recurrent cancers. This study evaluated 39 patients treated with PLDR re-irradiation from 2009 to 2016 at the same institution. These patients had a median follow-up time of 8.8 months (range 0.5-64.7). The median interval from the first radiation course and re-irradiation was 26.2 months; the median dose of the first and second course of radiation was 50.4 Gy and 50 Gy, respectively. Five patients (13\%) received concurrent systemic therapy. Of the 39 patients, 9 (23\%) developed grade $\geq 2$ acute toxicity, most commonly radiation dermatitis ( 5 of 9). None developed grade $\geq 4$ acute or subacute toxicity. The only grade $\geq 2$ late toxicity was late skin toxicity in 1 patient. On univariate analysis, toxicity was not significantly associated with the dose of the first course of radiation or re-irradiation, the interval to re-irradiation, or the reirradiation site. The local progression rate was $16.5 \%$ at 6 months and $23.8 \%$ at 12 months. Of the 41 disease sites for the 39 patients, 25 (61\%) were in the thoracic region including non-small cell lung and esophagus. For these thoracic patients 12 developed grade 1, 4 developed grade 2 and 4 developed grade 3 acute toxicities, and only 1 patient developed grade 1 late toxicity.

In the Tong X, et al. [73] PLDR study, 6 patients were treated with 3DCRT for recurrent primary lung or metastases. The prescription dose from the previous treatment ranged from 40 to 60 Gy and the prescription dose for the PLDR retreatment ranged from 30 to $60 \mathrm{~Gy}$. Good local control was achieved with 1 complete response and 5 partial responses. No acute toxicities were observed and only one patient developed grade 2 radiation pneumonitis.

\section{PLDR for pelvic cancers}

Paly J, et al [76] investigated pelvic PLDR re-irradiation using 3DCRT, IMRT and VMAT delivery techniques. They retrospectively analyzed a cohort of 38 patients treated at a single institution between June 2010 and July 2019. Median follow-up was 10.4 months (range: 1.3 to $86.6 \mathrm{mo}$ ). The tumor re-irradiation target site was $45 \%$ prostate, $21 \%$ rectal, $13 \%$ bladder, $8 \%$ gynecologic cancers, and $18 \%$ other. The median prior overlapping dose was 60 Gy EQD2 (range: 35 to $80 \mathrm{~Gy}$ ) for 31 external beam radiation patients with 
known initial fractionation. Four patients received initial LDR prostate brachytherapy (115 to 145 Gy). Initial RT was delivered at a median of 6.2 years (range: 1.0 to 23.9 y) before pelvic PLDR-RT. Overall, 37\% of patients received concurrent systemic therapy with PLDR re-treatment. Twenty-three patients were treated with definitive intent to a median re-irradiation PLDR-RT dose of 60 Gy (range: 50 to $76 \mathrm{~Gy}$ ). The one-year Kaplan-Meier local progression-free proportion based on clinical, biochemical, or radiographic response was $59 \%$ and 6 of 23 patients had no evidence of disease at their last follow-up. Among the 12 patients with available follow-up imaging, the best local tumor response showed complete response in $8 \%$, partial response in $42 \%$, stable disease in $25 \%$, and progressive disease in $25 \%$. Fifteen patients were treated palliatively with a median reirradiation PLDR-RT dose of 40 Gy (range: 28 to $60 \mathrm{~Gy}$ ). The one-year Kaplan-Meier local progression-free proportion based on clinical or radiographic response was $61 \%$. When available (15 patients), the best local tumor response on follow-up imaging showed a partial response in 33\%, stable disease in 53\%, and progressive disease in 13\%. Acute skin/ soft tissue grade 1 and 2 toxicity occurred in 5.3\% and 5.3\% of patients, respectively. Late skin/soft tissue grade 2 toxicity occurred in $2.6 \%$ of patients. Acute GU grade 1 and 2 toxicity occurred in $7.9 \%$ and $21.1 \%$ of patients, respectively. Late GU grade 1, 2, and 3 toxicity occurred in 13.2\%, 10.5\%, and $5.3 \%$ of patients, respectively. Acute GI grade 1 and 2 toxicity occurred in $7.9 \%$ and $7.9 \%$ of patients, respectively. Late GI grade 1,2 , and 3 toxicity occurred in $13.2 \%, 5.3 \%$, and $2.6 \%$ of patients, respectively. A sensitivity analysis evaluating late toxicity in patients with $>12$ months of follow-up demonstrated similar results. They concluded that PLDR and infrequent concurrent chemotherapy provided local control for the majority of patients for whom few if any, other options for local treatment existed. PLDR represents a viable option for patients in whom re-irradiation is otherwise high risk.

\section{PLDR for other clinical applications}

It is expected that PLDR will have other oncology applications besides the re-irradiation setting for recurrent cancers because of its potential local control and reduced normal tissue toxicity. In a recent retrospective study, Yan J, et al. [24] evaluated PLDR treatment of 22 patients with refractory malignancies. All patients previously received multiline treatments and failed with an estimated survival less than 6 months. Thus, palliative PLDR was given using conventional delivery techniques as described in reference [8]. Doses delivered by PLDR were between 48-60 Gy. Nine patients underwent PLDR for re-irradiation with cumulative doses between 104-166 Gy. The time interval from their last irradiation was 11 to 168 months. Ten patients received PLDR due to poor performance status. Three patients were given PLDR for bulky tumor. The irradiated sites included primary disease (seven patients), locally recurrent disease (nine patients), and retroperitoneal adenopathy (six patients). The median follow-up was 1 year (range 8-30 months). Overall, the 1-y local-regional control rate was $40 \%$. The 6-month survival rate was $76 \%$ and 1 -y survival rate was $69 \%$. For patients treated with PLDR re-irradiation, three patients (3/9) achieved partial remission and five patients (5/9) remained with stable disease at 1 month after the end of PLDR. A head and neck cancer patient remained progressionfree until the last follow-up, the LRRFS lasted for 16 months. A lung patient achieved complete response (CR) at 4 months after the PLDR, and the LRRFS lasted for 10 months. For patients treated with PLDR due to poor performance status, two patients (2/10) achieved partial remission 1 month after the PLDR. For the three patients given PLDR for bulky tumor, all of them achieved partial remission 1 month after the PLDR, and one patient achieved complete response at the fourth month. Overall, only 5 patients developed grade 3 or 4 toxicities (3 grade 3 bone marrow suppression, 1 grade 4 bone marrow suppression, and 1 grade 3 enteritis). No grade 5 toxicities occurred. All patients with toxicities recovered after treatments. They concluded that PLDR is an effective and safe option not only for re-irradiation but also for patients with poor performance status or bulky tumors.

More recent clinical trials at Fox Chase Cancer Center have extended to combination therapies of PLDR and chemotherapy for definitive treatment. A phase I study [77] investigates the role of PLDR for naive patients with nonsmall cell lung cancer or esophageal cancer whose planned treatment regimen is concurrent chemoradiotherapy followed by surgery. The total radiation dose is 50.4 Gy in daily fractions of 1.8 Gy for esophageal cancer and 60 Gy in daily fractions of 2 Gy for non-small cell lung cancer. For these patients, the rate of severe acute esophagitis during concurrent CRT is high (approximately 20\%) when conventional external beam radiation is utilized. Severe acute esophagitis can cause many adverse consequences such as severe discomfort, weight loss, hospitalization, interruption/early termination of treatment, and worse surgical complications for those who receive surgery after chemoradiotherapy. PLDR radiation has the potential to maintain the tumor control rates of conventional radiation while decreasing the toxicity to the surrounding normal 
tissue. Preliminary results of this clinical study have been reported by Price RA, et al. [78]. Data for 9 patients treated for esophageal cancer were evaluated based on the response of PET-defined tumor to PLDR radiotherapy to patients treated with conventional delivery using pre (scan-1) and post-treatment (scan-2) PET-CT scans. The mean pre to posttreatment percent volume reduction was $90.3 \%$ for PLDR and $74.7 \%$ for conventional delivery $(p=0.022)$. Volume reduction was also greater in node negative $(\mathrm{p}=0.037)$ and younger patients $(\mathrm{p}=0.065)$. The CR rate of PLDR was $33.3 \%$ vs. $16.7 \%$ for conventional delivery $(p=0.203)$. The average normalized mean SUV decrease from scan-1 to 2 for pre-treatment volumes was greater for PLDR delivery by approximately $22.8 \%$ (ns). This parameter for the posttreatment volume was greater for PLDR delivery by $6.8 \%$ (ns). These favorable results are indications suggestive of treatment effectiveness with PLDR.

Another phase I trial [79] investigates the safety and efficacy of PLDR radiation prior to surgery for non-metastatic pancreatic cancer. The prescription dose is escalated from the (lower) standard radiation dose of 56 Gy to a higher dose of 66 Gy for borderline resectable cases with the intent of facilitating a curative surgical intervention (e.g. providing more margin adjacent to non-resectable vessels and sterilizing regional lymph nodes). It is found that in pancreatic cancer models treated with regular radiation, fibroblasts in the microenvironment get more protumorigenic. However, with PLDR radiation, they do not get more protumorigenic. In fact, they revert back toward normal. It is expected that by treating with PLDR radiation, the pancreas is no longer supporting the cancer as much as it normally would.

\section{SUMMARY}

This paper reviewed the PLDR technique, the dosimetric requirements for PLDR treatment planning and dose delivery and the clinical applications of PLDR for cancer treatment. Outcomes from phase I clinical trials have shown favorable results of PLDR re-irradiation for treating recurrent cancers of various body sites. Further phase II and phase III clinical trials are warranted to quantify the therapeutic ratio of PLDR for different cancer types and determine its clinical efficacy. Other clinical applications of PLDR may be systematically investigated to fully exploit the potential benefits of LDHRS and low dose rate normal tissue repair for primary cancer treatment. In particular, PLDR can be used to treat bulky and/or resistant tumors, complex geometry that involves critical structures, and refractory patients to reduce normal tissue toxicities. Similarly, PLDR can be applied to early and intermediate stage cancer patients aiming at improvement in tumor control through dose escalation. PLDR can also play an important role in concurrent chemoradiotherapy to reduce the side effects of $\mathrm{RT}$ or combined toxicities of both modalities. Caution must be exercised, however, for those chemotherapy drugs that also cause cell DNA damage, which may change the transition dose for PLDR.

\section{REFERENCES}

1. Kavanagh BD, Timmerman RD. (2005). Stereotactic body radiation therapy, Lippincott Williams \& Wilkins, Philadelphia. PA. 1-153.

2. Buyyounouski MK, Balter P, Lewis B, D’Ambrosio DJ, Dilling TJ, et al. (2010). Stereotactic Body Radiotherapy for Early-Stage Non-Small-Cell Lung Cancer: Report of the ASTRO Emerging Technology Committee. Int J Radiation Oncology Biol Phys. 78:3-10.

3. Benedict SH, Yenice KM, Followill D, Galvin JM, Hinson W, et al. (2010). Stereotactic body radiation therapy: The report of AAPM Task Group 101. Med Phys. 37(8):4078101.

4. Ma CM. (2019). Physics and Dosimetric Principles of SRS and SBRT. Mathews J Cancer Sci. 4(2):22, DOI: https:// doi.org/10.30654/MJCS.10022.

5. Ma CM, Lomax T. Ed., Proton and carbon ion therapy. (Taylor \& Francis, New York, 2012).

6. Tomé WA, Howard SP. (2007). On the possible increase in local tumour control probability for gliomas exhibiting low dose hyper-radiosensitivity using a pulsed schedule. Br J Radiol. 80:32-37.

7. Adkison JB, Tome W, Seo S, Richards GM, Robins HI, et al. (2011) Reirradiation of large-volume recurrent glioma with pulsed reduced-dose-rate radiotherapy. Int J Radiat Oncol Biol Phys. 79(3):835-841.

8. Richards GM, Tome WA, Robins HI. (2009). Pulsed reduced dose-rate radiotherapy: a novel locoregional retreatment strategy for breast cancer recurrence in the previously irradiated chest wall, axilla, or supraclavicular region. Breast Cancer Res Treat. 114:307-313.

9. Ma CM, Luxton G, Orton C. (2011). Point-Counter-Point: Pulsed reduced dose rate radiation therapy is likely to become the treatment modality of choice for recurrent cancers. Med Phys. 38:4909-4911. 
10. Ma CM, Lin MH, Dai XF, Koren S, Klayton T, et al. (2012). Investigation of pulsed low dose rate radiotherapy using dynamic arc delivery techniques. Phys Med Biol. 57(14):4613-4626.

11. Favaudon V, Caplier L, Monceau V, Pouzoulet F, Sayarath $M$, et al. (2014). Ultrahigh dose-rate FLASH irradiation increases the differential response between normal and tumor tissue in mice. Sci Transl Med. 6:245-293.

12. Vozenin MC, Hendry JH, Limoli CL. (2019). Biological benefits of ultra-high dose rate FLASH radiotherapy: sleeping beauty awoken. Clin Oncol. 31: 407-415

13. Wilson JD, Hammond EM, Higgins GS, Petersson K. (2020). Ultra-High Dose Rate (FLASH) Radiotherapy: Silver Bullet or Fool's Gold? Front. Oncol. 9:1563. doi: 10.3389/fonc.2019.01563.

14. Rong Y, Paliwal B, Howard SP, Welsh J. (2011). Treatment Planning for Pulsed Reduced Dose-Rate Radiotherapy in Helical Tomotherapy. Int J Radiat Oncol Biol Phys. 79:934-942.

15. Lin MH, Price RA, Li JS, Kang SW, Li J, et al. (2013). Investigation of pulsed IMRT and VMAT for re-irradiation treatments: dosimetric and delivery feasibilities. Phys Med Biol. 58:8179-8196.

16. Kang SW, Lang J, Wang P, Li J, Lin MH, et al. (2014). Optimization strategies for pulsed low-dose-rate IMRT of recurrent lung and head and neck cancers. Journal of Applied Clinical Medical Physics. (15)3:102-113.

17. Li J, Lang J, Wang P, Kang S, Lin MH, et al. (2014). Intensity-modulated radiation therapy for pancreatic and prostate cancer using pulsed low-dose rate deliver techniques. Med Dosimetry. 39:330-336, S0958-3947.

18. Tyagi N, Yang K, Sandhu R, Yan D, Park SS, et al. (2013). External Beam Pulsed Low Dose Radiotherapy Using Volumetric Modulated Arc Therapy: Planning and Delivery. Medical Physics. 40:011704. https://doi. org/10.1118/1.4769119.

19. Murphy ES, Rogacki K, Godley A. (2017). Intensity modulated radiation therapy with pulsed reduced dose rate as a re-irradiation strategy for recurrent central nervous system tumors: An institutional series and literature review. Pract Radiation Oncol. 7:391-399.

20. Geurts M. (2017). TrueBeam Low Dose Rate Investigation for Pulsed Reduced Dose Rate IMRT. International Journal of Medical Physics. Clinical Engineering and Radiation Oncology. 6:139-149.
21. Mohindra P, Robins IH, Tomé WA, Hayes L, Howard SP. (2013). Wide-field Pulsed Reduced Dose Rate Radiotherapy (PRDR) for Recurrent Ependymoma in Pediatric and Young Adult Patients. Anticancer Res 33: 2611-2618.

22. Magnuson W, Robins HI, Mohindra P, Howard S. (2014). Large volume reirradiation as salvage therapy for glioblastoma after progression on bevacizumab. J Neurooncol. 117:133-139.

23. Lee CT, Dong Y, Li T, Freedman S, Anaokar J, et al. (2018). Local control and toxicity of external beam reirradiation with a pulsed low-dose-rate technique. Int J Radiat Oncol Biol Phys. 100:959-64.

24. Yan J, Yang J, Yang Y, Ren W, Liu J, et al. (2018). Use of pulsed low-dose rate radiotherapy in refractory malignancies. Transl Oncol. 11(1):175-181.

25. Rogacki K, Chao ST, Yu J, Godley A, Balagamwala E, et al. (2018). Review of Pulsed Reduced Dose Rate Reirradiation for Recurrent Tumors. J Cancer Clin Trials. 3:143. doi: 10.4172/2577-0535.10001.

26. Witt JS, Musunuru HB, Bayliss RA, Howard SP. (2019). Large volume reirradiation for recurrent meningioma with pulsed reduced dose rate radiotherapy. J Neurooncol. 141:103-109.

27. Burr AR, Robins HI, Bayliss RA, Howard SP. (2020). Pulsed Reduced Dose Rate for Reirradiation of Recurrent Breast Cancer. Practical Radiat Oncol. 10:e61-e70.

28. Joiner MC, Marples B, Lambin P, Short SC, Turesson I. (2001). Low-dose hypersensitivity: current status and possible mechanisms. Int J Radiat Oncol Biol Phys. 49(2):379-89.

29. Marples B, Collis SJ. (2008). Low-dose hyperradiosensitivity: past, present, and future. Int J Radiat Oncol Biol Phys. 70:1310-1318.

30. Wykes SM, Piasentin E, Joiner MC, Wilson GD, Marples B. (2006). Low-dose hyperradiosensitivity is not caused by a failure to recognize DNA double-strand breaks. Radiat Res. 165: 516-24.

31. Hall EJ, Brenner DJ. (1991). The dose-rate effect revisited: radiobiological considerations of importance in radiotherapy. Int J Radiat Oncol Biol Phys. 21:14031414. 
32. Dai X, Tao D, Wu H, Cheng J. (2009). Low dose hyperradiosensitivity in human lung cancer cell line A549 and its possible mechanisms. J Huazhong Univ Sci Technolog Med Sci. 29:101-6.

33. Singh B, Arrand JE, Joiner MC. (1994). Hypersensitive response of normal human lung epithelial cells at low radiation doses. Int J Radiat Biol. 65:457-464.

34. Short SC, Kelly J, Mayes CR, Woodcock M, Joiner MC. (2001). Low-dose hypersensitivity after fractionated low-dose irradiation in vitro. Int J Radiat Biol. 77:655664.

35. Harney J, Short SC, Shah N, Joiner M, Saunders MI. (2004). Low dose hyper-radiosensitivity in metastatic tumors. Int J Radiat Oncol Biol Phys. 59:1190-1195.

36. Krueger SA, Schoenherr D, Park S, Martines AA, Wilson GD, et al. (2010). Pulsed-protracted irradiation to overcome radioresistance. Int J Radiat Oncol Biol Phys. 78:S630.

37. Martin LM, Marples B, Lynch TH, Hollywood D, Marignol L. (2014). Exposure to low dose ionising radiation: Molecular and clinical consequences. Cancer Lett. 349:98-106.

38. Joiner MC, Lambin P, Malaise EP, Robson T, Arrand JE, et al. (1996). Hypersensitivity to very-low single radiation doses: Its relationship to the adaptive response and induced radioresistance. Mutat Res. 358:171-83.

39. Marples B, Joiner MC, Skov KA. (1994). The effect of oxygen on low dose hypersensitivity and increased radioresitance in Chinise hamster V79-379A cells. Radiat Res. 138(1 Suppl):S-17-20.

40. Xu B, Kim S, Lim D, Kastan MB. (2002). Two molecularly distinct $G(2) / M$ checkpoints are induced by ionizing irradiation. Mol Cell Biol. 22:1049-1059.

41. Short SC, Woodcock M, Marples B, Joiner MC. (2003). Effects of cell cycle phase on low-dose hyperradiosensitivity. Int J Radiat Biol. 79:99-105.

42. Krempler A, Deckbar D, Jeggo PA, Lobrich M. (2007). An imperfect G2M checkpoint contributes to chromosome instability following irradiation of S and G2 phase cells. Cell Cycle. 6:1682-1686.

43. Leonard BE. (2007). Thresholds and transitions for activation of cellular radioprotective mechanisms correlations between HRS/IRR and the "inverse" doserate effect. Int J Radiat Biol. 83:479-489.
44. Mitchell CR, Folkard M, Joiner MC. (2006). Effects of exposure to low-dose-rate 60Co gamma rays on human tumor cells in vitro. Radiat Res. 158:311-8.

45. Matsuya Y, McMahon SJ, Tsutsumi K, Sasaki K, Okuyama $\mathrm{G}$, et al. (2018). Investigation of dose-rate effects and cell-cycle distribution under protracted exposure to ionizing radiation for various dose-rates. Sci Rep. 8:1-14.

46. Steel GG. Basic clinical radiobiology. 3rd ed. (Oxford Univ Press Inc., New York, 2002) pp. 192-204.

47. Hall EJ, Giaccia AJ. (2006). Radiobiology for the radiologist. 6th ed. (Lippincott Williams \& Wilkins, Philadelphia, PA) pp 1-546.

48. Krueger SA, Collis SJ, Joiner MC, Wilson GD, Marples B. (2007). Transition in survival from low-dose hyper-radiosensitivity to increased radioresistance is independent of activation of ATM Ser1981 activity. Int J Radiat Oncol Biol Phys. 69(4):1262-1271.

49. Pierquin B, Calitchi E, Mazeron J, Piedbois P, Julien $M$, et al. (1985). Comparison between low dose rate radiotherapy and conventionally fractionated irradiation in moderately extensive cancers of the oropharynx. Int J Radiat Oncol Biol Phys. 11:431-9.

50. Wilson JF. (1978). Low dose rate teletherapy of unresectable carcinoma of the pancreas. Results of a pilot study. Proceedings of International Cancer Research workshop on low dose rate therapy. Am J Roentgenol. 131:1105-10.

51. Cooper SG, Cardew AP, Ferguson S, Joseph DJ, Hamilton CS, et al. (1990). Low dose rate teletherapy using telecaesium 137 unit radiobiological, physical and clinical considerations. Australas Radiol. 34:241-6.

52. Hamilton CS, Simpson SA, Ferguson S, Ostwald P, Hsu W, et al. (1993). Low dose rate teletherapy and tumour response. Australas Radiol. 37:210-2.

53. International Commission on Radiological Units and Measurements. (1985). Dose and Volume Specifications for Reporting Intracavitary Therapy in Gynecology. ICRU report 38 (ICRU).

54. Lin PS, Wu A. (2005). Not all 2 Gray radiation prescriptions are equivalent: Cytotoxic effect depends on delivery sequences of partial fractionated doses. Int J Radiat Oncol Biol Phys. 63:536-44.

55. Ma CM, Mu ZM, Tafo A, Chen L. (2010). Variation of cytotoxic effect with pulsed dose sequence and low dose rate radiation. Int J Radiat Oncol Biol Phys. 78:S629. 
56. Schoenherr D, Krueger SA, Martin L, Marignol L, Wilson GD, et al. (2013). Determining if low dose hyperradiosensitivity (HRS) can be exploited to provide a therapeutic advantage: A cell line study in four glioblastomamultiforme (GBM) cell lines. Int J Radiat Biol. 89(12):1009-16.

57. Terashima S, Hosokawa Y, Tsuruga E, Mariya Y, Nakamura T. (2017). Impact of time interval and dose rate on cell survival following low-dose fractionated exposures. Journal of Radiation Research. 58(6):782-790.

58. Dos Santos T, Liu S, Yang L, Li M, Chen L, et al. The optimal radiation dose rate for pulsed low-dose-rate radiation therapy using in vitro clonogenic analysis, AAPM ePoster Library. Dos Santos T. 07/12/20; 302744; PO-GeP-T-786.

59. Todorovic V, Prevc A, Zakelj MN, Savarin M, Bucek S, et al. (2020). Pulsed low dose-rate irradiation response in isogenic HNSCC cell lines with different radiosensitivity. Radiol Oncol. 54(2):168-179.

60. Park SS, Chunta JL, Robertson JM, Martinez AA, Oliver Wong CY, et al. (2011). MicroPET/CT imaging of an orthotopic model of human glioblastomamultiforme and evaluation of pulsed low-dose irradiation. Int J Radiat Oncol Biol Phys. 80:885-92.

61. Dilworth JT, Krueger SA, Dabjan M, Grills IS, Torma J, et al. (2013). Pulsed low-dose irradiation of orthotopic glioblastomamultiforme (GBM) in a pre-clinical model: effects on vascularization and tumor control. Radiother Oncol. 108:149-54.

62. Lee DY, Chunta JL, Park SS, Huang J, Martinez AA, et al. (2013). Pulsed versus conventional radiation therapy in combination with temozolomide in a murine orthotopic model of glioblastoma multiforme. Int J Radiat Oncol Biol Phys. 86(5):978-85.

63. Zhang P, Wang B, Chen X, Cvetkovic D, Chen L, et al. (2015). Local tumor control and normal tissue toxicity of pulsed low-dose rate radiotherapy for recurrent lung cancer: An in vivo animal study. Dose Response. 13(2): 1559325815588507.

64. Wang B, Ren J, Zhang Z, Cvetkovic D, Chen XM, et al. (2020). An In-Vivo Study on Pulsed Low-Dose-Rate Radiotherapy for Prostate Cancer. Mathews J Cancer Sci. 4(2):21. DOI:10.30654/mjcs.10021.

65. Meyer JE, Finnberg NK, Chen L, Cvetkovic D, Wang B, et al. (2017). Tissue TGF- $\beta$ expression following conventional radiotherapy and pulsed low-dose-rate radiation. Cell Cycle. 16:1171-4. doi:10.1080/15384101.2017.1317418.
66. Chan PK, Kuritzky, Zhang S, Tsai J, Li SD. (2008). Pulsed low dose-rate intensity modulated radiation therapy. Med Phys. 35(6):2741.

67. Price RA, Kuritzky N, Lin T, Ma CM. (2009). Pulsed reduced dose-rate intensity modulated radiotherapy (IMRT) delivery for use in the high dose re-irradiation setting. Med Phys. 36:2553.

68. Ma CM, Lin MH, Kang SW, Li J, Wang P, et al. (2014). Pulsed Low-Dose-Rate Radiation Therapy (PLDR) for Recurrent Cancers: Treatment Planning Strategies for IMRT and VMAT, IJROBP 90:s941 DOI:https://doi.org/10.1016/j. ijrobp.2014.05.2659.

69. Cannon GM, Tomé WA, Robins HI, Howard SP. (2007). Pulsed reduced dose-rate radiotherapy: case report: a novel re-treatment strategy in the management of recurrent glioblastoma multiforme. J Neurooncol. 83:307-311.

70. Li G-H, Liu Y, Tang J-L, Zhang D, Zhou P, et al. (2012). Pulsed reduced dose-rate radiotherapy as reirradiation for brain metastasis in a patient with lung squamouscelled carcinoma. Jpn J Clin Oncol. 42:856-860.

71. Bovi JA, Prah MA, Retslaff AA, Schmainda KM, Connelley JM, et al. (2020). Pulsed Reduced Dose Rate Radiotherapy in Conjunction With Bevacizumab or Bevacizumab Alone in Recurrent High-grade Glioma: Survival Outcomes. Int J Radiation Oncol Biol Phys. 108:979-986.

72. Li G-H, Zhu B, Yang F, Ma CK, Yang D-Q. (2012). Use of cetuximab in combination with pulsed reduced dose-rate radiotherapy in a patient with recurrence of nasopharyngeal carcinoma in the neck, Experimental and Therapeutic Medicine. 3:869-872.

73. Tong X, Luo F, Liu Y, Zhang W, Xu Q et al. (2015). Pulsed Low Dose Rate RT for Recurrent Cancers: A Clinical Observation, IJROBP. 93(3): E478. DOI: 10.1016/j. ijrobp.2015.07.1767.

74. Ma CM, Meyer JE (Principal Investigators). (2011). Phase I Study of Pulsed Low Dose Rate Reirradiation Delivered with 3DCRT/IMRT for Palliation of Recurrent Tumors, Fox Chase Cancer Center, IRB\#11-044/OER-RT-042. Clinical Trials gov Identifier: NCT01470365.

75. Meyer JE, Galloway TJ, Ruth K, Ross EA, Buyyounouski $\mathrm{MK}$, et al. (2016). Efficacy of pulsed low-dose-rate reirradiation: results from a phase 1 clinical trial. Int J Radiat Oncol Biol Phys. 96:S147. 
76. Paly J, Deng M, Lee CT, Hayes SB, Galloway TJ, et al. (2020). Pelvic Reirradiation Utilizing Pulsed Low-dose Rate Radiation Therapy. Am J Clin Oncol. 43:748-751. doi: 10.1097/COC.0000000000000741.

77. Meyer JE (Principal Investigator). (2017). Pulsed Low Dose Rate Radiation With Concurrent Chemotherapy for Non-Small Cell Lung Cancer and Esophageal Cancer, Fox Chase Cancer Center, IRB\# 16-1051/OER-RT-101. Clinical Trials gov Identifier: NCT03094884.

78. Price RA, Meyer JE, Hallman MA, Yu JQM, Gou J, et al. (2019). Pre- and Post-treatment PET-CT Image Analysis of Pulsed-low-dose-rate (PLDR) Compared to Conventional RT Delivery for Initial Treatment of Esophageal Tumors, IJROBP 105: E797. DOI:https://doi. org/10.1016/j.ijrobp.2019.06.2483.
79. Meyer JE (Principal Investigator). (2020). Utilizing Pulsed Low-Dose-Rate (PLDR) Radiation to Prevent de novo stromal Activation; a Neoadjuvant Pancreatic Adenocarcinoma Phase I Trial, Fox Chase Cancer Center, IRB\# 18-1085/OER-RT-155. Clinical Trials gov Identifier: NCT04452357. 